\title{
Research Note \\ Particle acceleration in thick parallel shocks with high compression ratio
}

\author{
J. J. P. Virtanen ${ }^{1}$ and R. Vainio ${ }^{2}$ \\ 1 Tuorla Observatory, Väisälä Institute for Space Physics and Astronomy, Väisäläntie 20, 21500 Piikkiö, Finland \\ e-mail: joni.virtanen@utu.fi \\ 2 Department of Physical Sciences, PO Box 64, 00014 University of Helsinki, Finland \\ e-mail: rami.vainio@helsinki.fi
}

Received 2 May 2005 / Accepted 1 June 2005

\begin{abstract}
We report studies on first-order Fermi acceleration in parallel modified shock waves with a large scattering center compression ratio expected from turbulence transmission models. Using a Monte Carlo technique we have modeled particle acceleration in shocks with a velocity ranging from nonrelativistic to ultrarelativistic and a thickness extending from nearly steplike to very wide structures exceeding the particle diffusion length by orders of magnitude. The nonrelativistic diffusion approximation is found to be surprisingly accurate in predicting the spectral index of a thick shock with large compression ratio even in the cases involving relativistic shock speeds.
\end{abstract}

Key words. acceleration of particles - shock waves - cosmic rays

\section{Introduction}

First-order Fermi acceleration in shocks with steplike velocity profile is well known to be an efficient way of producing nonthermal particle populations with power law (see, e.g., reviews of Drury 1983; Kirk \& Duffy 1999, for nonrelativistic and relativistic studies, respectively). In the nonrelativistic regime with infinitely thin step-shocks this mechanism produces particle momentum distributions $f(p) \propto p^{-s}$ with spectral index $s$ depending only on the compression ratio of the shock, $r$, as $s=3 r /(r-1)$. For the relativistic case also the shock speed $V_{1}$ affects, and the spectral index - in the case of isotropic pitchangle diffusion - can be written as

$s_{\mathrm{KW}}=\frac{3 V_{1} c^{2}-2 V_{1} V_{2}^{2}+V_{2}^{3}}{\left(V_{1}-V_{2}\right) c^{2}}=\frac{3 r}{r-1}\left(1-\frac{V_{1}^{2}(2 r-1)}{3 r^{2} c^{2}}\right)$

as was recently shown by Keshet \& Waxman (2005).

In a modified shock - i.e., in a shock with a nontrivial velocity profile and finite shock thickness - the acceleration efficiency drops as the thickness increases (e.g., Schneider \& Kirk 1989; Virtanen \& Vainio 2003). Again, in the nonrelativistic case the resulting spectral index as a function of shock thickness and compression ratio can have a simple analytical solution, found by Drury et al. (1982), whereas the matter becomes much more complicated in the relativistic regime (e.g., Schneider \& Kirk 1989). In their nonrelativistic study
Drury et al. (1982) showed that while the produced spectral index tends to the well known step-shock limit when the shock thickness approaches zero, the high energy part of the spectrum can have a power law even when the transition is large compared to free path of an accelerating particle. The produced spectral index depends on the thickness of the transition region and the compression ratio as

$s=\frac{3 V_{1}}{V_{1}-V_{2}}\left(1+\frac{1}{\beta} \frac{V_{2}}{V_{1}-V_{2}}\right)=\frac{3 r}{r-1}\left(1+\frac{1}{\beta(r-1)}\right)$,

where $\beta$ is a parameter inversely proportional to the shock thickness. For a self-consistent cosmic-ray dominated shock it has the value of $\beta=\frac{1}{2}\left(1+\gamma_{\mathrm{c}}\right)$, where $\gamma_{\mathrm{c}}$ is the adiabatic index of the cosmic-ray gas. Later Schneider \& Kirk (1989) found the nonrelativistic diffusion approximation with spectral index of the form of Eq. (2) to agree well even in cases in which the thickness of the shock transition is of the order of the particle mean free path where the diffusion approximation should not be mathematically justified. In the relativistic regime the diffusion approximation was, expectedly, found to fail to approximate the produced spectral index. One should also note that Drury et al. (1982) and Schneider \& Kirk (1989) neglected the second-order Fermi acceleration in their studies. The effect of this mechanism in the shock downstream has been studied analytically in modified nonrelativistic shocks by Schlickeiser (1989) and numerically in relativistic shocks by 
Virtanen \& Vainio (2005), and it leads to more complex dependence of the produced spectral index of the shock's Peclet and Alfvénic Mach number and compression ratio.

From Eq. (2) it becomes obvious that even shocks with large thickness (i.e., small $\beta$ ) can accelerate particles efficiently if only the compression ratio is sufficiently high. This may seem irrelevant, as the gas compression ratio of a shock is generally limited to rather small values, e.g., $r<\left(\gamma_{\mathrm{c}}+1\right) /\left(\gamma_{\mathrm{c}}-1\right)$ for cosmic-ray dominated nonrelativistic shocks. However, the compression ratio felt by the accelerated particles is not that of the flow; as already noted by Bell (1978), one needs to consider the finite phase speed of the hydromagnetic waves responsible for particle scattering around the shock waves. Taking the fluctuations to be Alfvén waves, the problem of turbulence transmission at the shock is tractable and the effective scattering center compression ratio is found to be much larger than the gas compression ratio for shocks of low Alfvénic Mach number (Vainio \& Schlickeiser 1998, 1999; Vainio et al. 2003, 2005).

\section{Scattering-center compression ratio}

As shown by Vainio \& Schlickeiser (1998) for nonrelativistic and Vainio et al. (2003, 2005) for relativistic speeds, shocks with low to moderate quasi-Newtonian Alvénic Mach number $M \gtrsim \sqrt{r}$ transmit the Alfvén waves from upstream to downstream so that most of the downstream waves propagate antiparallel to the direction of the shock-frame plasma flow. Thus, the scattering center compression ratio, calculated assuming a vanishing upstream cross-helicity,

$r_{k}=\frac{V_{1}\left(1+H_{\mathrm{c} 2} V_{\mathrm{A} 2} V_{2} / c^{2}\right)}{V_{2}+H_{\mathrm{c} 2} V_{\mathrm{A} 2}}$

tends to infinity as the Alfvénic Mach number of the shock $M=u_{1} / u_{\mathrm{A} 1}$ (where $u_{1}$ and $u_{\mathrm{A} 1}$ are the shock proper speed and the upstream proper Alfvén speed respectively, both measured in the rest frame of the upstream plasma) approaches the critical Mach number $M_{\mathrm{c}}=\sqrt{r}$, since $V_{2} \rightarrow V_{\mathrm{A} 2}$ and $H_{\mathrm{c} 2} \rightarrow-1$ at this limit. Here, $V_{\mathrm{A} 2}$ and $H_{\mathrm{c} 2}$ are the Alfvén speed and the wave cross helicity in the downstream region, and they can be calculated from the shock jump conditions. This is shown in Fig. 1 for parallel shocks with three velocities assuming a momentum independent scattering mean free path and a vanishing upstream cross-helicity. For details on how to compute $r_{k}$, see Vainio et al. (2003, 2005).

The scattering hydromagnetic fluctuations have wavelengths comparable to the Larmor radius of the resonant particles. Thus, for shock waves discussed in this paper, the Alfvén waves actually see the shock front as a thick structure. Thus, their transmission becomes governed by the WKB theory. A detailed analysis of this case is beyond the scope of this short Note but qualitatively one expects wave transmission in these shocks to show very similar behavior as in steplike shock waves: antiparallel propagating waves are amplified much more than the parallel propagating ones because of the conservation of the wave action, $\left(V \pm V_{\mathrm{A}}\right)^{2} P^{ \pm}(f, x) / V_{\mathrm{A}}=$ const., where $f=\left(V \pm V_{\mathrm{A}}\right) k / 2 \pi$ and $P^{+(-)}(f, x)$ are the (conserved) wave frequency and the power spectrum of the parallel

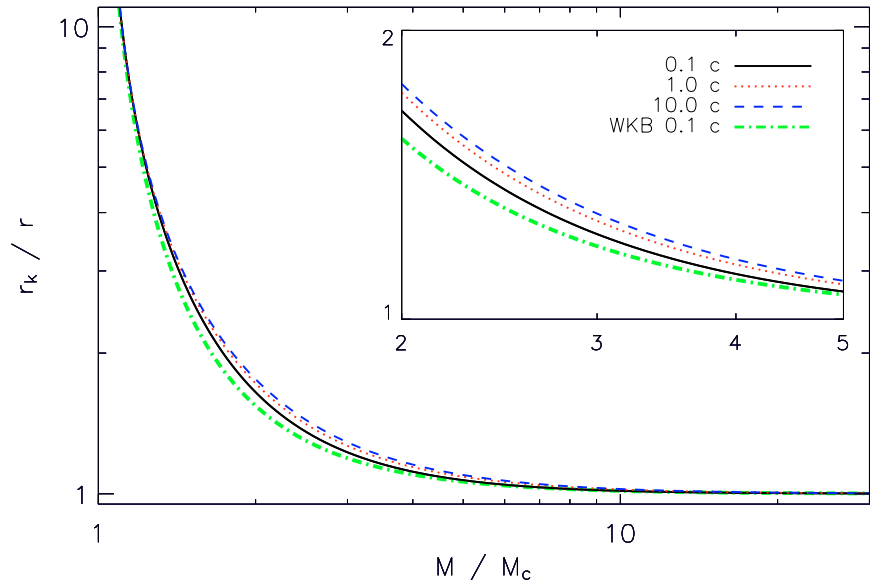

Fig. 1. Ratio of the scattering center compression ratio $r_{k}$ to that of the gas $r$ for different Alvénic Mach numbers $M$. The spectral index of the turbulence power spectrum is $q=2$, corresponding to a momentumindependent particle mean free path. $M_{\mathrm{c}}=\sqrt{r}$. See text for details.

(antiparallel) propagating waves, respectively. For the nonrelativistic case and for spectral index $q$ of the fluctuations one finds after a short calculation

$I_{2}^{ \pm}(k)=I_{1}^{ \pm}(k) r^{q+1 / 2} \frac{(M \pm 1)^{q+1}}{\left(M \pm r^{1 / 2}\right)^{q+1}}$

for the downstream intensities of waves propagating parallel (antiparallel) to the flow, $I_{1[2]}^{+(-)}$. For a vanishing upstream cross helicity we get

$H_{\mathrm{c} 2}=\frac{(M+1)^{q+1}(M-\sqrt{r})^{q+1}-(M-1)^{q+1}(M+\sqrt{r})^{q+1}}{(M+1)^{q+1}(M-\sqrt{r})^{q+1}+(M-1)^{q+1}(M+\sqrt{r})^{q+1}}$.

Thus, $r_{k} \rightarrow \infty$ as $M \rightarrow \sqrt{r}$, as in our earlier calculations. An example of this is shown in Fig. 1 for nonrelativistic shock (dash-dot-dashed line, labeled "WKB $0.1 c$ "). Of course, the singularity is not reached in practice because the wave pressure in the downstream region becomes large and makes the compression ratio stay below $M^{2}$ in a self-consistent calculation (Vainio \& Schlickeiser 1999). Nevertheless, large scattering compression ratios are expected for low-Mach number shocks even for large shock thickness.

\section{Particle acceleration}

We have used test-particle Monte Carlo simulations to calculate the particle energy distribution $N(E) \propto E^{-\sigma}$ spectral indices $\sigma=s-2$ resulting from first-order Fermi acceleration at the shock for parallel shocks. Detailed description of the code is given elsewhere (Appendix A of Virtanen \& Vainio 2005). The simulations follow test-particles in a one dimensional flow. Small pitch angle scatterings are performed in the frame comoving with the scattering centers. Instead of using two scattering wave fields, $I^{ \pm}$, we calculate the effective wave speed as $H_{\mathrm{c}} V_{\mathrm{A}}$ and apply only a single scattering wave field propagating at this speed relative to the plasma. This eliminates stochastic acceleration from the model and allows us to study the effect of first-order Fermi acceleration at the shock, only. 
We use the hyperbolic tangent function of Drury et al. (1982) for the scattering center speed (denoted hereafter by $V$ ) profile across the shock:

$V(x)=V_{1}-\frac{V_{1}-V_{2}}{2}\left[1+\tanh \left(\frac{3 \beta\left(V_{1}-V_{2}\right)}{2 c} \frac{x}{\lambda}\right)\right]$.

Here, $\lambda$ is the (constant) particle mean free path and the parameter $\beta$ the same as in Eq. (2). In this study we consider $\beta$ as a free parameter and simulate shocks of different widths by varying its value. Defining the diffusion length of the particles in the usual nonrelativistic manner as $d_{i}=\frac{1}{3} \lambda c / V_{i}$, we can write the inverse shock thickness as $W^{-1}=\frac{1}{2} \beta\left(d_{1}^{-1}-d_{2}^{-1}\right)$. Thus, nonrelativistic simulations with $\beta \ll 1$ correspond to shocks with a thickness much larger than the diffusion length of the accelerated particles.

For each simulation we fix the proper speed of the shock relative to the upstream scattering centers, $u_{1}=\Gamma_{1} V_{1}=c\left(\Gamma_{1}^{2}-1\right)^{1 / 2}$, and the (scattering center) compression ratio $r_{k}=V_{1} / V_{2}$. Particles are given a small initial energy and they are injected into the shock in the upstream so that they have time to isotropize before reaching the shock transition region. Particles are then traced in the shock until they hit the downstream escape boundary at $x_{2}$. This boundary is set sufficiently far away from the shock so that the particles have enough time to isotropize in the rest frame of the downstream scattering centers. A probability of return is calculated and applied for each particle crossing the boundary; the method is described e.g., by Ellison et al. (1990). Particle splitting is used to improve the statistics at high energies. Energy losses due to synchrotron emission are not considered in the simulations.

Simulations were run for shock proper speeds $u_{1}=$ $\{0.1,1,10\} c$, compression ratios $r_{k}=\{3,10,30\}$, and for $\beta$ having values between $\sim 0.07$ and $\sim 1000$. The resulting spectral indices are shown in Fig. 2 together with the predictions of the nonrelativistic diffusion approximation from Eq. (2).

In the nonrelativistic case $\left(V_{1} \approx u_{1}=0.1 c\right)$ the results are as expected: spectral indices are in accordance with the diffusion approximation in all cases, even where the shock thickness is comparable to the diffusion length of the particles. This is the case with all compression ratios studied. For the mildly relativistic case $\left(u_{1}=1.0 c\right.$, i.e., $V_{1} \approx 0.7 c$ and $\Gamma_{1} \approx 1.4$ ) and the lowest compression ratio $r_{k}=3$ (corresponding to the gas compression ratio of a relativistic parallel shock) the simulated indices start to differ from the diffusion approximation, and the differences grow even larger for the ultrarelativistic case $\left(u_{1}=10 c\right.$ with $V_{1} \approx 0.995 c$ and $\left.\Gamma_{1} \approx 10\right)$. At the step-shock limit, where $\beta \rightarrow \infty$, the index from the ultrarelativistic shock tends to $\sigma \approx 2.2$ as expected, and also the $u_{1}=c$ case produces indices harder than the diffusion theory predicts. This, of course, is expected from previous studies. As the shock thickness increases ( $\beta$ decreases) behavior similar to that reported by Schneider \& Kirk (1989) is seen: while for thin shocks the diffusion approximation predicts harder spectra than those obtained from simulation, for shocks with wider transition the spectra are softer than the values from the diffusion approximation.

The most interesting result of our study is, however, that as the compression ratio increases above the traditional

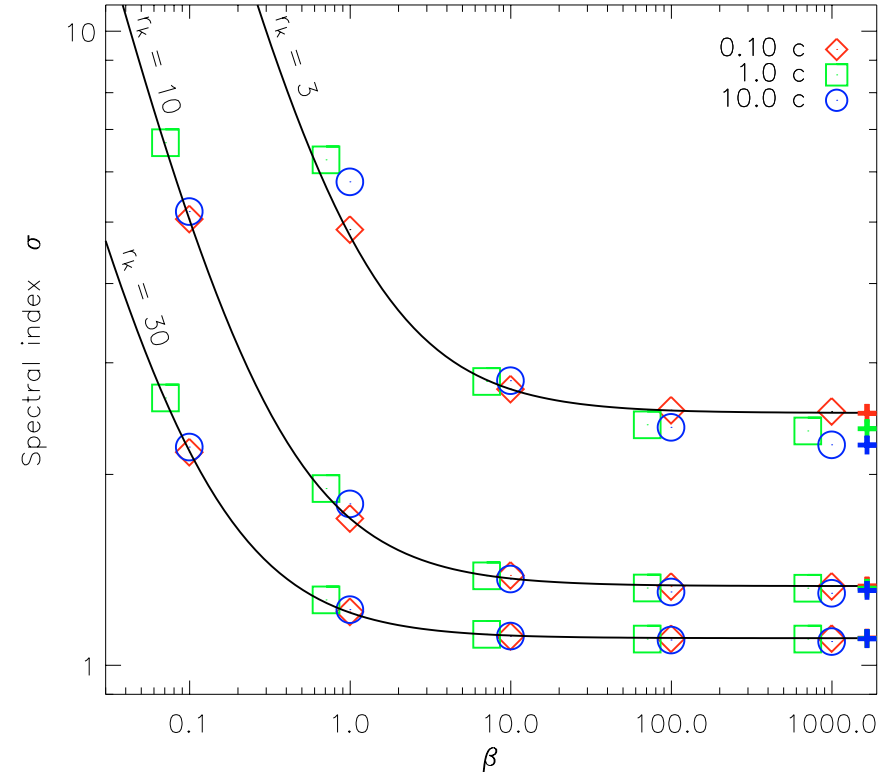

Fig. 2. Particle energy spectral indices as a function of $\beta$ for shock proper speeds $u_{1}=0.1 c$ (diamonds), $1.0 c$ (squares), and $10.0 c$ (circles) for scattering center compression ratios $r_{k}=3,10$, and 30; the crosses at the right end denote values obtained from Eq. (1) for step shocks $(\beta \rightarrow \infty)$. Solid lines show the predictions of the nonrelativistic diffusion approximation. The statistical errors of the fitted spectral indices are of the order of 0.040 or less for all datapoints.

gas-compression ratio values, the nonrelativistic diffusion approximation works rather well even for relativistic shocks. Small deviations are seen in all cases where relativistic effects are present, but the spectral indices can be approximated by Eq. (2) to an accuracy of the relevant observations, especially for the case $r_{k}=30$. Furthermore, even shocks with very wide transition can produce spectra that are hard, compared to those usually considered to be produced in parallel shocks.

\section{Conclusions}

Based on our analysis, we arrive at the following conclusions of particle acceleration at modified shocks.

I. Alfvén-wave transmission can lead to large scattering center compression ratios in parallel shocks regardless of the thickness of the shock front if the Alfvénic Mach number of the shock is close to the critical value $M_{\mathrm{c}}=\sqrt{r}$.

II. Modified parallel shocks can accelerate particles efficiently even if their thicknesses exceed the diffusion length of the accelerated particles, if the scattering center compression ratio is large.

III. Nonrelativistic theory of particle acceleration in modified shocks, Eq. (2), gives a good approximation to the spectral index at shocks with large scattering center compression ratio even for ultrarelativistic shock velocities.

Acknowledgements. CSC, the Finnish IT center for science, is acknowledged for providing part of the computing facilities. 


\section{References}

Bell, A. R. 1978, MNRAS, 182, 147

Drury, L. O'C. 1983, Rep. Prog. Phys., 46, 973

Drury, L. O'C., Axford, W. I., \& Summers, D. 1982, MNRAS, 198, 833

Ellison, D. C., Jones, F. C., \& Reynolds, S. P. 1990, ApJ, 360, 702

Keshet, U., \& Waxman, E. 2005, Phys. Rev. Lett., 94, 111102

Kirk, J. G., \& Duffy, P. 1999, J. Phys. G, 25, R163

Schlickeiser, R. 1989, ApJ, 336, 264
Schneider, P., \& Kirk, J. G. 1989, A\&A, 217, 344

Vainio, R., \& Schlickeiser, R. 1998, A\&A, 331, 793

Vainio, R., \& Schlickeiser, R. 1999, A\&A, 343, 303

Vainio, R., Virtanen, J. J. P., \& Schlickeiser, R. 2003, A\&A, 409, 821

Vainio, R., Virtanen, J. J. P., \& Schlickeiser, R. 2005, A\&A, 431, 7

Virtanen, J. J. P., \& Vainio, R. 2003, in High Energy Blazar Astronomy, ed. L. O. Takalo, \& E. Valtaoja, ASP Conf. Proc., 299, 143

Virtanen, J. J. P., \& Vainio, R. 2005, ApJ, 621, 313 